\title{
Eficacia de la cirugía laparoscópica radical para mejorar la dismenorrea secundaria en endometriosis grado III y IV
}

\author{
Efficacy of radical laparoscopic surgery for dysmenorrhea \\ relief in patients with stage III and IV endometriosis Gamaliel Rodríguez Reyes, ${ }^{\star}$ Javier Benítez Beltrán*
* Alta Especialidad de Cirugía Endoscópica Ginecológica en el Hospital «Lic. Adolfo López Mateos», Instituto de Seguridad y Servicios Sociales de los Trabajadores del Estado (ISSSTE). México.
${ }^{\ddagger}$ Facultad Mexicana de Medicina de la Universidad La Salle. México. \\ Roberto Ignacio Montiel Mora, ${ }^{*, \neq}$ Javier Contreras Orozco, ${ }^{*, \neq}$ Fabiola del Carmen Brito Sandoval, ${ }^{*, \neq}$
}

\section{RESUMEN}

Objetivo: Evaluar la eficacia de la cirugía laparoscópica radical para reducir la severidad de dismenorrea en pacientes con endometriosis grados III y IV. Diseño: Estudio de cohorte prospectivo. Material y métodos: Se comparó la gravedad de dismenorrea prequirúrgica versus el puntaje a los tres meses posquirúrgicos, utilizando la escala visual análoga (EVA) en 64 mujeres con endometriosis grados III y IV, a las cuales se les realizó cirugía laparoscópica radical. Resultados: La edad promedio de diagnóstico de dismenorrea fue de $32.9 \pm 7.7$ años y la edad a la que se realizó la intervención quirúrgica fue de $35.6 \pm 6.5$ años, el $35.9 \%$ de las mujeres tuvieron como hallazgos quirúrgicos endometriosis grado III y el $64 \%$ de ellas con este padecimiento en grado IV. El sangrado transquirúrgico promedio fue de $155.6 \pm 3.3 \mathrm{~mL}$ y los días de estancia intrahospitalaria fueron $2.09 \pm$ 0.66 , no se reportaron complicaciones transquirúrgicas. La media de la puntuación de la EVA para la severidad de la dismenorrea prequirúrgico fue de $8.25 \pm 0.89$ y el puntaje a los tres meses posquirúrgicos fue de 2.06 \pm 1.06 , el cual fue significativamente menor ( 8.25 versus 2.06 puntos, $p=$ 0.001 ), el porcentaje en que se redujo el puntaje de la EVA prequirúrgica versus la posquirúrgica fue de $74.4 \%$. Conclusión: La cirugía laparoscópica radical, en la mayoría de las pacientes con endometriosis grados III y IV, mejora la dismenorrea en más de $70 \%$. Este beneficio se observa principalmente a los tres meses posquirúrgicos.

Palabras clave: Endometriosis, dismenorrea, cirugía radical laparoscópica.

\section{ABSTRACT}

Objective: To evaluate the efficacy of radical laparoscopic surgery for dysmenorrhea relief in patients with stages III and IV endometriosis. Study design: Prospective cohort. Material and methods: Using the visual analogue pain scale (VAS), the severity of dysmenorrhea before surgery is compared with VAS score three months after surgery, in sixty-four women diagnosed with endometriosis stages III and IV, treated with radical laparoscopic surgery. Results: The average age of endometriosis diagnosis was $32.9 \pm 7.7$ years and the mean age when the surgery was performed was $35.6 \pm 6.5$ years, $35.9 \%$ of the women had stage III endometriosis surgical findings and $64 \%$ of them had stage IV endometriosis. The average trans-surgical bleeding was $155.6 \pm 3.3 \mathrm{~mL}$ and the number of days of hospital stay were $2.09 \pm$ 0.66 . The mean VAS score for the severity of presurgical dysmenorrhea was $8.25 \pm 0.89$ and the 3-month postsurgical score was $2.06 \pm 1.06$, which was significantly lower ( 8.25 vs 2.06 points, $p=0.001$ ), the score of the presurgical VAS versus the postsurgical VAS was reduced by a percentage of $74.4 \%$. Conclusions: Laparoscopic surgery may offer relief or improvement of more than $70 \%$ in the majority of patients with dysmenorrhea secondary to stages III and IV endometriosis. These benefits seem greater postoperatively after three months.

Keywords: Endometriosis, dysmenorrhea, laparoscopic radical surgery.

Recibido: 22/10/2020. Aceptado: 27/12/2020

Correspondencia: Roberto Ignacio Montiel Mora

E-mail: robemora71@gmail.com

Citar como: Montiel MRI, Contreras OJ, Brito SFC, Rodríguez RG, Benítez BJ. Eficacia de la cirugía laparoscópica radical para mejorar la dismenorrea secundaria en endometriosis grado III y IV. Rev Mex Cir Endoscop. 2020; 21 (3): 145-148. https://dx. doi.org/10.35366/99838 


\section{INTRODUCCIÓN}

La endometriosis se define como la presencia de tejido endometrial fuera de la cavidad uterina y es una de las principales causas de dolor pélvico crónico en mujeres en edad reproductiva, se ha reportado hasta en $32 \%$ de las mujeres con dolor pélvico crónico intervenidas quirúrgicamente. ${ }^{1}$ En algunos casos la dolencia es severa y puede afectar la calidad de vida. ${ }^{2}$ El tratamiento óptimo de esta enfermedad aún es controversial, la elección del método debe ser individualizado, dependiendo de la severidad de los síntomas, la extensión y localización de las lesiones, la edad, así como el deseo reproductivo., ${ }^{3,4}$

El tratamiento médico es efectivo pero en muchos casos insuficiente y es un abordaje temporal, ya que al suspender el medicamento la recurrencia es frecuente, del mismo modo, los efectos adversos son poco tolerados por algunas mujeres jóvenes. Un procedimiento eficaz que ha cobrado relevancia en la actualidad es la cirugía, la remoción amplia de todas las lesiones endometriósicas, adherenciolisis y restauración de la anatomía, conformando una opción para aliviar el dolor y preservar la fertilidad. ${ }^{5}$

En la última década, los avances de la intervención laparoscópica han identificado lesiones endometriósicas atípicas que anteriormente no se conocían, han permitido un mejor enfoque anatómico, escisión completa y amplia de endometriosis, lo que convierte a dicho abordaje en la primera línea de tratamiento quirúrgico. ${ }^{6} \mathrm{La}$ cirugía laparoscópica debe ser radical, ya que la escisión incompleta de focos endometriósicos se asocia con recurrencia temprana de la sintomatología; sin embargo, este abordaje es técnicamente difícil, requiere cirujanos con amplia experiencia y se puede asociar con complicaciones intestinales y vesicales. ${ }^{7,8}$

Se configuró esta cohorte de tipo prospectivo a fin de evaluar la eficacia de la cirugía laparoscópica radical para disminuir la severidad de la dismenorrea secundaria a endometriosis grados III y IV, sin incrementar la tasa de complicaciones perioperatorias.

\section{MATERIAL Y MÉTODOS}

Se analizaron 70 mujeres con dismenorrea de moderada a severa, refractaria a tratamiento médico. Todas fueron sometidas a cirugía laparoscópica, de marzo 2018 a febrero 2019. Durante la operación fueron catalogadas según la American Society for Reproductive Medicine (ASRM), ${ }^{9} 23$ casos se clasificaron como endometriosis grado III, 41 con grado IV y seis sin hallazgos de endometriosis grados I y II, estas mujeres fueron excluidas del estudio.
La cirugía laparoscópica radical incluía adherenciolisis, cistectomía, restauración de la anatomía y escisión de nódulos fibrosos y lesiones endometriósicas profundas.

En las 64 mujeres con endometriosis grados III y IV incluidas en el análisis se valoró la severidad de la dismenorrea prequirúrgica y, posteriormente, a los tres meses posquirúrgicos con la escala visual análoga (EVA). Un puntaje de 1-5 se consideró dolor leve, 6-7 puntos dolor moderado y 8-10 puntos dolencia severa. Una disminución de al menos dos puntos entre la EVA prequirúrgica y EVA del tercer mes se consideró una mejoría en la seriedad de la dismenorrea.

El análisis estadístico se realizó con el programa SPSS, versión 23.0 (SPSS INC, Chicago, Illinois). Las variables continuas se reportarán con media y desviación estándar, las variables categóricas como frecuencias y porcentajes, se utilizará t de Student para diferencias de medias y prueba de $\chi^{2}$ para diferencia de proporciones entre ambos grupos. La significancia estadística se consideró cuando $\mathrm{p}<0.05$.

\section{RESULTADOS}

De las 64 pacientes con dismenorrea moderada a severa que fueron intervenidas por cirugía laparoscópica, 35.9\% (23) de ellas tenían endometriosis grado III y $64 \%$ (41) grado IV. La edad de diagnóstico de dismenorrea en ambos grupos fue de 24 a 52 años (promedio $32.9 \pm 7.7$ años) y la edad de tratamiento quirúrgico fue de 20 a 52 años (promedio $35.6 \pm 6.5$ años). El sangrado transquirúrgico promedio fue de $155.6 \pm 3.3 \mathrm{~mL}$ y los días de estancia intrahospitalaria fueron $2.09 \pm 0.66$, no se reportaron complicaciones transquirúrgicas (Tabla 1).

La media del puntaje de la EVA para la severidad de la dismenorrea prequirúrgica fue de $8.25 \pm 0.89$ y la puntuación a los tres meses posquirúrgicos fue de $2.06 \pm 1.06$, la cual fue significativamente menor $(p=0.001)$ en mujeres con endometriosis grados III y IV (Figura 1).

El porcentaje en que se redujo el puntaje de la EVA prequirúrgico versus el posquirúrgico fue de $74.4 \%$. La mayoría de las mujeres, $60.9 \%$ (39), tuvieron una disminución de la puntuación de EVA prequirúrgica versus posquirúrgica de $51-79 \%$. Solamente el $6.2 \%$ (cuatro) tuvieron mejoría de $100 \%$ (Tabla 2).

\section{Tabla 1: Características basales $(\mathrm{N}=64)$.}

\begin{tabular}{lcc} 
& Media \pm DE & Rango \\
\hline Edad diagnóstico (años) & $32.9 \pm 7.7$ & $24-52$ \\
Edad cirugía (años) & $35.6 \pm 6.5$ & $20-52$ \\
Sangrado (mL) & $155.6 \pm 3.3$ & $50-400$ \\
Estancia hospitalaria (días) & $2.09 \pm 0.66$ & $1-4$ \\
\hline
\end{tabular}




\section{DISCUSIÓN}

La cirugía laparoscópica es una opción terapéutica dirigida a mujeres con falla del tratamiento médico para disminuir la severidad de la dismenorrea. Algunos estudios reportan que el procedimiento laparoscópico reduce el dolor pélvico crónico, mejora el índice de fertilidad y acorta el periodo de espera del embarazo..$^{6-8}$ El tratamiento radical se ha reportado como efectivo en el mejoramiento del malestar y la calidad de vida; sin embargo, este abordaje no está estandarizado, por lo que se utilizan diferentes métodos de tratamiento para la escisión de lesiones endometriósicas profundas como láser, energía bipolar, etcétera. Existe poca información acerca de la extensión que debe tener la escisión de las lesiones para mejorar el dolor y la recurrencia. ${ }^{10,11}$

Después de 12 meses de seguimiento, Ford y colaboradores reportan mejoría en dismenorrea del $61 \%{ }^{10}$ con resección radical de endometriosis. En general se reporta mejoría del dolor de $60-84 \%$ a los 29 meses de seguimiento. ${ }^{12}$ En este estudio, la cirugía laparoscópica radical mejoró la gravedad de la dismenorrea más del $70 \%$ en la mayoría de las pacientes con endometriosis grados III y IV. Este beneficio se observó a los tres meses de seguimiento, se necesitan estudios prospectivos o ensayos clínicos a fin de valorar si esta mejoría en la severidad de la dismenorrea continuará o incrementará después de más tiempo de seguimiento.

Los principales riesgos de la cirugía radical son las lesiones rectales y vesicales. Donnez y su grupo reportaron perforación rectal en $1.4 \%$ y ureteral en $0.8 \%$ de 500 procedimientos laparoscópicos. ${ }^{6}$ En ninguna de las operaciones realizadas se notificaron complicaciones intraoperatorias o posoperatorias y ninguna de las pacientes tuvo que ser reintervenida por recurrencia del dolor a los tres meses.

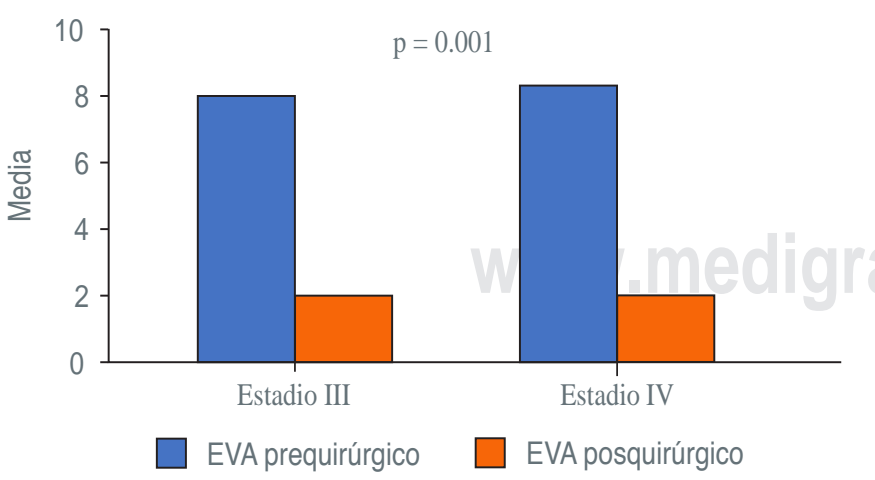

Figura 1: La media del puntaje de la EVA para valorar la severidad de la dismenorrea prequirúrgica fue $8.25 \pm 0.89$ y el puntaje a los tres meses posquirúrgicos fue $2.06 \pm 1.06(p=0.001)$.

\begin{tabular}{cc}
$\begin{array}{c}\text { Tabla 2: Mejoría EVA prequirúrgica } \\
\text { versus EVA posquirúrgica. }\end{array}$ \\
\hline$\%$ Mejoría & $\mathrm{n}(\%)$ \\
\hline 100 & $4(6.2)$ \\
$80-99$ & $16(25)$ \\
$51-79$ & $39(60.9)$ \\
$<50$ & $5(7.8)$ \\
\hline
\end{tabular}

\section{CONCLUSIONES}

La cirugía laparoscópica radical en la mayoría de las pacientes con endometriosis grados III y IV mejora la dismenorrea. Este procedimiento requiere de cirujanos entrenados para poder ser efectuada de forma segura.

\section{REFERENCIAS}

1. Becker CM, Laufer MR, Stratton P, Hummelshoj L, Missmer SA, Zondervan KT et al. World endometriosis research foundation endometriosis phenome and biobanking harmonisation project: I. Surgical phenotype data collection in endometriosis research. Fertil Steril. 2014; 102: 12131222.

2. Haas D, Oppelt P, Shebl O, Shamiyeh A, Schimetta W, Mayer R. Enzian classification: does it correlate with clinical symptoms and the rASRM score? Acta Obstet Gynecol Scand. 2013; 92: 562-566.

3. Jones GL, Kennedy SH, Jenkinson C. Health-related quality of life measurement in women with common benign gynecologic conditions: a systematic review. Am J Obstet Gynecol. 2002; 187: 501-511.

4. Johnson NP, Hummelshoj L; World Endometriosis Society Montpellier Consortium. Consensus on current management of endometriosis. Hum Reprod. 2013; 28: 1552-1568.

5. Kennedy S, Bergqvist A, Chapron C, D'Hooghe T, Dunselman G, Greb R et al. ESHRE guideline for the diagnosis and treatment of endometriosis. Hum Reprod. 2005; 20: 2698-2704.

6. Donnez J, Squifflet J, Pirard C, Jadoul P, Wyns C, Smets $M$. The efficacy of medical and surgical treatment of endometriosis-associated infertility and pelvic pain. Gynecol Obstet Invest. 2002; 54 Suppl 1: 2-7; discussion 7-10.

7. Darai E, Dubernard G, Coutant C, Frey C, Rouzier R, Ballester M. Randomized trial of laparoscopically assisted versus open colorectal resection for endometriosis: morbidity, symptoms, quality of life, and fertility. Ann Surg. 2010; 251: 1018-1023.

8. Porpora MG, Pallante D, Ferro A, Crisafi B, Bellati F, Benedetti Panici P. Pain and ovarian endometrioma recurrence after laparoscopic treatment of endometriosis: a long-term prospective study. Fertil Steril. 2010; 93: 716-721. 
9. Guzick DS, Silliman NP, Adamson GD, Buttram VC Jr, Canis M, Malinak LR et al. Prediction of pregnancy in infertile women based on the American Society for Reproductive Medicine's revised classification of endometriosis. Fertil Steril. 1997; 67: 822-829.

10. Ford J, English J, Miles WA, Giannopoulos T. Pain, quality of life and complications following the radical resection of rectovaginal endometriosis. BJOG. 2004; 111: 353-356.
11. Duepree HJ, Senagore AJ, Delaney CP, Marcello PW, Brady KM, Falcone T. Laparoscopic resection of deep pelvic endometriosis with rectosigmoid involvement. J Am Coll Surg. 2002; 195: 754-758.

12. Meuleman C, Tomassetti C, D'Hooghe TM. Clinical outcome after laparoscopic radical excision of endometriosis and laparoscopic segmental bowel resection. Curr Opin Obstet Gynecol. 2012; 24: 245-252. 